\title{
Pharmacokinetics and Pharmacodynamics of Antimicrobial Agents in Burn Patients
}

\author{
Kaitlin A. Pruskowski ${ }^{1,2}$
}

\begin{abstract}
Background: Infection is common after burn injury and accounts for the most frequent complications of burn injury. This review describes the effects of burn injury on pharmacokinetics, focusing on the impact of these changes on antimicrobial therapy.

Methods: The published literature on pharmacokinetics and pharmacodynamics in burn injury of antibiotic use was reviewed.

Results: Physiologic and metabolic changes of burn injury can alter pharmacokinetic parameters, leading to larger volumes of distribution, faster hepatic metabolism, and increased renal clearance. Changes in pharmacokinetics may lead to subtherapeutic doses of commonly used antibiotic and antifungal agents.

Conclusions: Although not all antimicrobial agents are well studied in the burn population, dose recommendations exist for some agents. Additional research is needed to cover the systemic antimicrobial agents used in the care of burn patients so that appropriate dosing adjustments can be made to optimize outcomes.
\end{abstract}

Keywords: antimicrobials; burn; pharmacodynamics; pharmacokinetics

$\mathbf{I}_{\mathbf{2}}$ NFECTION IS COMMONLY ENCOUNTERED after burn injury and is a leading cause of death in this patient population [1,2]. Between 2009 and 2018, infections (to include pneumonia, urinary tract infection, cellulitis, wound infection, bacteremia, and septicemia) accounted for six of the 10 most common complications after burn injury [3]. Dysregulation of immune function and burn patients' inflammatory response are altered after burn injury, making recognition of infection challenging. Although infections and sepsis may present differently in the burn population, antibiotic treatment principles remain the same. Because of physiologic and metabolic alterations after burn injury, pharmacokinetic parameters of antimicrobials may be altered, and changes to antimicrobial dosing may need to be made. This review describes the effects of burn injury on pharmacokinetic and pharmacodynamic parameters, specifically in relation to antibiotic and antifungal therapy.

\section{Pharmacokinetics Overview}

To understand how burn injury and critical illness affect pharmacokinetics, one must first understand the basic pharmacokinetic parameters [4-6]. Pharmacokinetics (PK) de- scribes the movement the absorption, distribution, metabolism, and elimination of medications from the body, whereas pharmacodynamics (PD) describes the response that results when a drug reaches its target receptor or site of action. Often, these terms are used together.

Absorption, distribution, metabolism, and elimination, also known as ADME, are the four basic pharmacokinetic parameters. Absorption is the process by which a medication reaches the systemic circulation from its site of administration. The bioavailability $(\mathrm{F})$ of a medication is the fraction of the medication that reaches the systemic circulation.

Distribution describes the process by which drug molecules move throughout the vasculature into the organs and tissues. The volume of distribution $(\mathrm{Vd})$ describes the relation between a dose of a medication and the resulting serum concentration. Drugs that are lipophilic will have a high Vd, whereas drugs that are hydrophilic will have a low Vd.

Metabolism is the conversion of drug molecules to active or inactive metabolites. Typically, the clinician thinks of this as occurring in the liver.

Elimination is the removal of drug from the body. Typically, the clinician thinks of this occurring via the kidneys. The elimination rate constant, ke, describes the fraction of

\footnotetext{
${ }^{1}$ U.S. Army Institute of Surgical Research, Fort Sam Houston, Texas, USA.

${ }^{2}$ Uniformed Services University School of the Health Sciences, F. Edward Hébert School of Medicine, Bethesda, Maryland, USA.
} 
drug in the body eliminated per unit time. Alternatively, drug clearance, CL, can be used to describe elimination, and shows the volume of serum or blood completely cleared of the drug per unit time.

\section{Pharmacokinetic Changes in Burn Patients}

During the first 48 to 72 hours after initial injury, patients with severe thermal injury may present in burn shock and will undergo large-volume fluid resuscitation. Inflammation, both at the site of injury and systemically, causes vasodilation, vascular hyperpermeability, and decreases in interstitial oncotic pressure, shifting fluid and albumin out of the intravascular space to the interstitial space [7]. This, coupled with massive fluid resuscitation, lead to increases in the Vd. Larger than normal doses may need to be administered to achieve a therapeutic serum concentration [8].

During this time, patients also have decreased myocardial contractibility and cardiac output, decreasing blood flow to the gut, the liver, and the kidneys. In turn, the rate and extent of absorption of enterally administered medications may be decreased, leading to a delay in the onset of action and decreases in maximum serum concentrations [7]. Decreases in hepatic metabolism and renal elimination may also occur, and less-frequent dosing may be needed to avoid adverse drug effects [8].

By day five post-injury, patients start to develop a postburn hypermetabolic response. This response can be sustained for up to one to three years after burn injury [8]. During the hypermetabolic phase, systemic inflammation and oxidative stress lead to increased cardiac contractility and increased cardiac output. For enterally administered medications, absorption from the gut may be faster, shorting the time to onset of action. Blood flow to the liver and kidneys are also increased, increasing hepatic metabolism and renal clearance.

It is thought that sustained increases in catecholamines, glucorticoids, proinflammatory cytokines (tumor necrosis factor- $\alpha$, interleukin- $1 \beta$, and interleukin-6, among others) contribute to the hypermetabolic response $[9,10]$. Changes in glucocorticoid levels after burn injury can modulate the expression of cytochrome P450 (CYP450) enzymes, resulting in changes in drug metabolism [11,12]. Increases in interleukin-6 have been associated with changes in the CYP450 system, specifically in decreases in CYP450 3A4 [13]. However, the exact changes in CYP450 have not been studied extensively in the burn population and remain unknown at this time.

Increased cardiac output increases blood flow to the kidneys and can potentially lead to renal elimination of medications. Also, increases in catecholamines during the postburn hypermetabolic response, specifically norepinephrine, can lead to increases in creatinine clearance, and potentially drug clearance [14]. There may be additional physiologic changes to increased, or augmented, renal clearance, however, these mechanisms are not well understood at this time. Additionally, patients experience non-renal clearance of drugs via exudate leakage from partial and full-thickness burns [7]. During this phase, burn patients may need more frequent dosing of medications to maintain therapeutic serum concentrations. One cannot forget that these patients also will be globally volume-overloaded (although not necessarily intravascularly volume overloaded) and will have an increased Vd. Thus, higher than usual doses may need to be administered more frequently to maintain optimal serum concentrations.

It is important to note that recovery from burn injury is a dynamic process. Critically ill burn patients will fluctuate between resuscitative and hypermetabolic phases throughout their hospital stay. Clinicians will need to evaluate their patients' day-to-day clinical picture and be mindful how this will affect changes in PK/PD. Patients in the hypermetabolic phase of burn injury may require higher doses or more frequent dosing of antimicrobial agents. The next section explores the impact of burn injury on specific antibiotic and antifungal agents.

\section{Pharmacokinetic Considerations by Antimicrobial Agent}

\section{Beta-Lactams}

Beta-lactam antibiotic agents, including penicillins, cephalosporins, and carbapenems, exert bactericidal activity by inhibiting cell wall synthesis. The PD of this class of antibiotic agents is optimized when the serum concentration of the antibiotic is above the minimum inhibitory concentration (MIC) for at least 50\%

Penicillins. Piperacillin-tazobactam is the most-studied penicillin in the burn population. A study by Olbrisch et al. [15] showed that piperacillin/tazobactam dosed at $4.5 \mathrm{~g}$ intravenously three times daily failed to achieve optimal piperacillin concentrations in burn and other critically ill patients. Augmented renal clearance, often seen in burn patients during the hypermetabolic phase, was directly related to low plasma concentrations of piperacillin. Olbrisch et al. [15] postulated that high doses of piperacillin, up to $8 \mathrm{~g}$ four times daily, infused over three hours would be needed to optimize PK/PD. A review by Cota et al. [7] showed that when given via extended infusion (four-hour infusion), piperacillin-tazobactam $4.5 \mathrm{~g}$ intravenously given every six hours optimized PK/PD parameters and was effective in treating most susceptible organisms.

Cephalosporins. Infections caused by gram-negative organisms, specifically Pseudomonas aeruginosa, are not uncommon after burn injury, and the third-generation cephalosporins, ceftazidime and cefepime, are often used for treatment. Serum concentrations of ceftazidime are subtherapeutic after usual doses, with concentrations being up to $43 \%$ lower in burn patients than healthy patients. It has been recommended to administer doses up to $6 \mathrm{~g}$ per day via extended infusions to optimize PK/PD [7,16]. Similarly, it has been recommended that cefepime also be administered via prolonged infusion (up to four hours) [7].

Although newer agents, including ceftaroline, ceftazidime-avibactam, ceftolozane-tazobactam, and cefidericol, are alternatives for treating infections caused by multi-drug-resistant organisms, these agents have not been studied in the burn population; it is unknown how burn injury effects the PK/PD of these agents. Dose adjustments for these agents cannot be recommended at this time.

Carbapenems. Carbapenems include doripenem, ertapenem, imipenem, and meropenem. The pharmacokinetics of 
imipenem and meropenem have been studied in burn patients. The available literature shows that PK parameters of these agents are not substantially altered in patients with burn injury [16]. However, for infections caused by organisms with a MIC greater than $2 \mathrm{mcg} / \mathrm{mL}$, extended infusions (administered over two to three hours) may be needed to optimize the PD of these agents $[7,17]$.

The new carbapenem/ $\beta$-lactamase combination, meropenem-vaborbactam, has not yet been studied specifically in burn patients. Pharmacokinetic changes and the ideal dosing strategy for burn patients is not known at this time.

\section{Aztreonam}

Aztreonam is classified as a monobactam and exerts its activity by inhibiting bacterial cell wall synthesis. Patients infected with Pseudomonas isolates with a high MIC ( $>8$ $\mathrm{mcg} / \mathrm{mL}$ ), extended infusions of aztreonam may be required. Alternatively, aztreonam may need to be given more frequently (every six hours) [7]. However, for most infections, the usual dose of aztreonam can be utilized.

\section{Aminoglycosides}

Aminoglycosides inhibit bacterial protein synthesis by binding to the $30 \mathrm{~S}$ ribosomal subunit. These agents have concentration-dependent killing; achieving a peak concentration of at least eight to 10 times the MIC of the infecting organism provides the most bactericidal activity. Patient with severe burn injury often have an increased Vd and higher than expected doses may needed to achieve the goal peak, Usually, these agents are dosed in a weight-based fashion, using ideal body weight. Recent studies have shown that weight-based dosing of gentamicin and tobramycin, dosed at $7 \mathrm{mg} / \mathrm{kg}$ actual body weight and $10 \mathrm{mg} / \mathrm{kg}$ actual body weight respectively, can achieve peak concentrations greater than $20 \mathrm{mcg} / \mathrm{mL}[18,19]$.

The aminoglycosides also exert a post-antibiotic effect; the bactericidal activity of these agents may last several hours after serum concentrations become undetectable. During the hypermetabolic phase after burn injury, renal function is often augmented, and the aminoglycosides are removed from the body faster; increased dosing frequency may be needed. Because of these PK changes, extended interval (once-daily) dosing is unlikely to optimize the PD of these agents. Therapeutic drug monitoring should be performed frequently and dose or frequency adjustments should be made to achieve optimal bactericidal potential and avoid prolonged periods of undetectable drug concentrations. [7]

\section{Fluoroquinolones}

The fluoroquinolones include ciprofloxacin, levofloxacin, moxifloxacin, and delafloxacin. This class of antibiotic agents inhibits DNA gyrase, preventing the unwinding and transcription of bacterial DNA. These agents are optimized when the area under the curve (AUC) to MIC ratio is $>125: 1$. For burn patients with preserved or augmented renal function, ciprofloxacin dosed at $400 \mathrm{mg}$ intravenously three times daily or levofloxacin dosed at $750 \mathrm{mg}$ intravenously daily achieves this AUC:MIC ratio [7,20,21]. However, dose adjustments must be made for patients with renal insufficiency.
The newer fluoroquinolones, moxifloxacin and delafloxacin, have not yet been studied in burn patients. No dose or frequency modifications can be recommended at this time.

\section{Polymyxins}

The polymyxins include colistin (polymyxin E) and polymyxin B. These antibiotic agents re-emerged as options for the treatment of infections caused by multi-drug-resistant gram-negative organisms. The polymyxins agents are detergents, and damage bacterial cytoplasmic membrane to cause bacterial cell death.

Colistin is administered as a prodrug, colistin methanesulfonate (CMS), also known as colistimethate. Colistin methanesulfonate is hydrolyzed to the active form of colistin, and it is estimated that only $30 \%$ of CMS is hydrolyzed to the active form in healthy patients [22]. It is unknown how burn injury affects the metabolism of CMS to colistin.

The PK/PD properties of colistin and how burn injury affects these properties are still being studied. Achieveing an AUC:MIC ratio of greater than 30:1 causes the greatest bactericidal effect. It has been suggested that a goal steadystate plasma colistin concentration of $2 \mathrm{mcg} / \mathrm{mL}$ may be reasonable to treat infections caused by organisms with an MIC less than $1 \mathrm{mcg} / \mathrm{mL}$ [23]. A recent case series showed the $\mathrm{Vd}$ of colistin is increased in burn patients, leading to lower plasma concentrations [24]. Percent total body surface area (TBSA) burned was affected by colistin's minimum and maximum concentrations, as well as AUC. Thus, patients with severe burn injury may require increased doses to optimize colistin's PK/PD. Exposure to high doses of colistin comes with an increased risk of nephrotoxicity. However, the exact dosing regimen of CMS in burn patients remains unknown. Until the PK/PD changes of colistin are better understood and dosing recommendations can be made, singleagent therapy with colistin should not be used [7]. Polymyxin $\mathrm{B}$ has not extensively studied in burn patients, and no dose adjustments can be recommended at this time.

\section{Vancomycin}

Vancomycin exerts its bactericidal activity by binding to the D-ala-D-ala portion of the cell wall precursor, inhibiting bacterial cell wall synthesis. This glycopeptide antibiotic covers most gram-positive organisms, including methicillinresistant Staphylococcus aureus (MRSA). Vancomycin is often included in empiric antibiotic therapy for MRSA coverage. Because of physiologic alterations associated with burn injury, patients tend to have increased $\mathrm{Vd}$ and faster elimination of vancomycin. A recent study showed that larger percent TBSA, shorter time from injury, and higher creatinine clearance (as predicted by the Cockcroft-Gault equation) are all associated with faster CL of vancomycin [25].

Until recently, it was recommended to optimize the PD properties of vancomycin by targeting a trough of 15 to 20 $\mathrm{mcg} / \mathrm{mL}$. Guidelines published in 2020 now recommend targeting an AUC:MIC ratio of 400-600:1 to optimize PD without increasing the risk of nephrotoxicity [26]. Because of the novelty of these guidelines, it is unclear how vancomycin dosing in burn patients will be impacted under the new guidelines Previous literature has shown that targeting a trough between 15 and $20 \mathrm{mcg} / \mathrm{mL}$ is likely to produce an AUC:MIC ratio of at least 360:1 [27]. Previous studies have 
shown that burn patients may require $40-70 \mathrm{mg} / \mathrm{kg}$ per day of vancomycin to achieve ideal PK/PD. Some patients may require more than $70 \mathrm{mg} / \mathrm{kg}$ per day, and will require vancomycin continuous infusions to attain a therapeutic vancomycin levels [7]. Because large doses of vancomycin may be required, one must balance the risks and benefits of vancomycin and must be prepared to perform frequent therapeutic drug monitoring.

\section{Linezolid}

Linezolid is an oxazolidinone antibiotic that inhibits bacterial protein synthesis by binding to the $50 \mathrm{~S}$ ribosomal subunit and is used to treat infections caused by grampositive organisms, including MRSA and vancomycinresistant enterococcus (VRE). It is bacteriostatic, whereas other options for the treatment of MRSA are bacteriocidal. Because of this, it is not the treatment of choice for bacteremia. In order to optimize the PD of linezolid, the AUC:MIC ratio should be greater than 80:1 [7]. Previous literature showed that compared with patients without burn injury, the AUC may be decreased up to $50 \%$ in patients who are severely burned [28]. A recent study showed the linezolid dosed at $600 \mathrm{mg}$ intravenously every eight hours may optimize the PK/PD of linezolid in burn patients. [29]

\section{Daptomycin}

Daptomycin binds to components of bacterial cell membranes and causes rapid depolarization, thereby inhibiting the synthesis of bacterial DNA, RNA, and proteins. The spectrum of activity of daptomycin includes gram-positive organisms, including MRSA. A drawback of daptomycin is that its agent is inactivated by surfactant in the lungs, thus, it is not a treatment option for pneumonia. Daptomycin had dosedependent activity, but there are newer theories that optimizing the AUC:MIC ratio may yield better outcomes. A PK study showed that compared with non-burn patients, burn patients had decreased AUC, increased VD, and increased CL of daptomycin [30]. The authors concluded that a daily dose of $12 \mathrm{mg} / \mathrm{kg}$ of daptomycin may be needed to optimize PK/PD. A recent study verified that daptomycin $12 \mathrm{mg} / \mathrm{kg}$ daily optimized the PK/PD of daptomycin without causing serious adverse effects.

\section{Antifungal Agents}

Because of the nature of their injury coupled with critical illness, patients with severe burn injury are at high risk for developing fungal infections. Usually, these infectious develop several weeks after burn injury and carry a high rate of mortality [31]. However, there is limited literature on PK/PD changes of antifungal agents in burn patients.

\section{Azoles}

The azole antifungal agents include fluconazole, posaconazole, voriconazole, and isavuconazole (available as the prodrug isavuconazonium).

Posaconazole, voriconazole, and isavuconazole are options for treating invasive fungal wound infectious caused by molds, because these agents cover Aspergillus, Fusarium, and Mucor species. Of these agents, only voriconazole has limited literature available. A small case series showed
Table 1. Antimicrobial Dosing Recommendations

\begin{tabular}{|c|c|}
\hline Agent & Dosing recommendations \\
\hline $\begin{array}{l}\text { Piperacillin- } \\
\text { tazobactam }\end{array}$ & $4.5 \mathrm{~g}$ IV q6h infused over $4 \mathrm{~h}$ \\
\hline Ceftazidime & $2 \mathrm{~g}$ IV $\mathrm{q} 8 \mathrm{~h}$ infused over $3 \mathrm{~h}$ \\
\hline Cefepime & $2 \mathrm{~g}$ IV q8h infused over $4 \mathrm{~h}$ \\
\hline $\begin{array}{l}\text { Imipenem- } \\
\text { cilastatin }\end{array}$ & $500 \mathrm{mg}$ IV q6h ${ }^{\mathrm{a}}$ \\
\hline Meropenem & $1-2 \mathrm{~g} \mathrm{IV} \mathrm{q} 8 \mathrm{~h}^{\mathrm{a}}$ \\
\hline Aztreonam & $2 \mathrm{~g}$ IV q8h infused over $3 \mathrm{~h}$ OR $2 \mathrm{~g}$ IV q6h \\
\hline Gentamicin & $\begin{array}{l}7 \mathrm{mg} / \mathrm{kg} \text { (actual body weight) IV } \mathrm{q} 24 \mathrm{~h} \\
\text { (may need more frequent dosing, } \\
\text { therapeutic drug monitoring recommended) }\end{array}$ \\
\hline Tobramycin & $\begin{array}{l}10 \mathrm{mg} / \mathrm{kg} \text { (actual body weight) IV q24h } \\
\text { (may need more frequent dosing, } \\
\text { therapeutic drug monitoring recommended) }\end{array}$ \\
\hline Ciprofloxacin & $400 \mathrm{mg}$ IV q8h \\
\hline Levofloxacin & 750 mg IV q24h \\
\hline Vancomycin & $\begin{array}{l}40-70 \mathrm{mg} / \mathrm{kg} \text { per day to achieve } \\
\text { AUC:MIC ratio of } 400-600: 1 \\
\text { (therapeutic drug monitoring recommended) }\end{array}$ \\
\hline Linezolid & $600 \mathrm{mg}$ IV q8h \\
\hline Daptomycin & $12 \mathrm{mg} / \mathrm{kg}$ IV daily \\
\hline Micafungin & $\begin{array}{l}100 \mathrm{mg} \text { IV daily } \\
\text { (may need higher doses for high-MIC } \\
\text { Candida spp. or Aspergillus }\end{array}$ \\
\hline
\end{tabular}

$\mathrm{IV}=$ intravenous $; \mathrm{AUC}=$ area under the curve $\mathrm{MIC}=$ minimum inhibitory concentration.

${ }^{\mathrm{a}}$ Infuse over $3 \mathrm{~h}$ for infecting organisms with MIC $>2 \mathrm{mcg} / \mathrm{mL}$.

that there was large intra-patient variability of the PK parameters of voriconazole in critically ill burn patients. Despite this, PK/PD targets were met for all patients included [32]. Because there is a paucity of literature for these agents, dose adjustment recommendations cannot be made at this time.

\section{Echinocandins}

The echinocandins, caspofungin, micafungin, and anidulafungin, have concentration-dependent activity with postantifungal effects. These agents inhibit $1,3-\beta$-D-glucan synthase, affecting the formation of fungal cell walls and are active against Candida and some Aspergillus species. Only micafungin has been studied specifically in the burn population. A daily dose of $100 \mathrm{mg}$ of micafungin has been shown to have decreased peak concentrations and increased elimination in critically ill burn patients [33,34]. However, micafungin dosed at $100 \mathrm{mg}$ daily was sufficient to achieve PK/ PD targets for Candida species with an MIC of $8 \mathrm{mcg} / \mathrm{mL}$ or less and for Candida parapsilosis with an MIC of $64 \mathrm{mcg} / \mathrm{mL}$ or less [35]. Doses higher than $100 \mathrm{mg}$ daily may need to be considered to optimize PK/PD and achieve optimal serum levels in patients who are infected with Candida who have higher MICs or Aspergillus. Little data are available on micafungin doses greater than $100 \mathrm{mg}$ daily, thus high-dose micafungin cannot be recommended at this time.

\section{Amphotericin}

Amphotericin binds to ergosterol and alters the permeability of the fungal cell membrane, leading to fungal cell death. Amphotericin has activity against a broad-spectrum 
of fungi, including Candida, Aspergillus, and Mucor, among others. Amphotericin is often used to treat invasive fungal wound infections. Often, the liposomal formulation is preferred to decrease the risk of nephrotoxicity. Because of the lack of available literature on PK/PD changes of amphotericin in burn patients, dose adjustments cannot be recommended.

Table 1 summarizes the above dosing recommendations. All recommendations are for patients with preserved or augmented renal function. For patients with renal insufficiency, dose adjustments should be made as per the antimicrobial's package insert.

\section{Conclusion}

Bacterial and fungal infections are not uncommon after burn injury. Physiologic and metabolic changes after severe burns may alter the PK of antibiotic and antifungal agents. Dosing strategies may need to be altered to optimize the PK and PD of these agents. Physiologic changes that affect PK and $\mathrm{PD}$ be considered when designing antimicrobial dosing regimens in the burn population.

\section{Acknowledgments}

The opinions or assertions contained herein are the private views of the authors and are not to be construed as official or as reflecting the views of the Department of the Army or the Department of Defense.

\section{Funding Information}

No funding was received.

\section{Author Disclosure Statement}

No competing financial interests exist.

The opinions or assertions contained herein are the private views of the authors, and are not to be construed as official or as reflecting the views of the Department of the Army or the Department of Defense.

\section{References}

1. Church D, Elsayed S, Reid O, et al. Burn wound infections. Clin Micro Rev 2006;19:403-434.

2. Gomez R, Murray CK, Hospenthal DR, et al. Causes of mortality by autopsy findings of combat casualties and civilian patients admitted to a burn unit. J Am Coll Surg 2009;208:348-354.

3. American Burn Association. National Burn Repository 2019 Update: Report of Data from 2009-2018. Chicago, IL: 2019.

4. Boucher BA Wood GC, Swanson JM Pharmacokinetic changes in critical illness. Crit Care Clin 2006;22:255-271.

5. Bauer LA. Clinical pharmacokinetic and pharmacodynamics concepts. In: Applied Clinical Pharmacokinetics, 3rd ed. Weitz M, and Pancotti R (eds). New York, NY: McGraw Hill Medical; 2001:3-27.

6. Smith BS, Yogaratnam D, Levasseur-Franklin KE, et al. Introduction to drug pharmacokinetics in the critically ill patient. Chest 2012;141:1327-1338.

7. Cota J, Fakhri Ravari A, Rowan MP, et al. Intravenous antibiotic and antifungal agent pharmacokineticpharmacodynamic dosing in adults with severe burn injury. Clin Ther 2016;39:2016-2031.

8. Blanchet B, Jullien V, Vinsonneau C, Tod M. Influence of burns on pharmacokinetics and pharmacocdynamics of drugs used in the care of burn patients. Clin Pharmacokinet 2008;47:635-654.

9. Jeschke MG, Gauglitz G, Kulp GA, et al. Long-term persistence of the pathophysiologic response to severe burn injury. PLoS ONE 2011;6:e21245.

10. Auger C, Samadi O, Jeschke MG. The biochemical alterations underlying post-burn hypermetabolism. Biochim Biophys Acta 2017;1863(10 Pt B):2633-2644.

11. Dvorak Z, Pavek P. Regulation of drug-metabolizing cytochrome P450 enzymes by glucocorticoids. Drug Met Rev 2010;42:621-635.

12. Matoulkova P, Pavek P, Maly J, Vicek J. Cytochrome P450 enzyme regulation by glucocorticoids and consequences in terms of drug interaction. Expert Opin Drug Metab Toxicol 2014;10:425-435.

13. Haas CE, Kaufman DC, Jones CE, et al. Cytochrome P450 3A4 activity after surgical stress. Crit Care Med 2003;31: 1338-1346.

14. Udy AA, Roberts JA, Boots RJ, et al. Augmented renal clearance: Implications for antibacterial dosing in the critically ill. Clin Pharmacokinet 2010;49:1-16.

15. Olbrish K, Kisch T, Thern J, et al. After standard dosage of piperacillin plasma concentrations of drug are subtherapeutic in burn patients. Arch Pharmacol 2019;392:229-241.

16. Weinbren MJ. Pharmacokinetics of antibiotics in burn patients. J Antimicrob Chemother 1999;44:319-327.

17. Corcicone S, D'Avolio A, Loia RC, et al. Pharmacokinetics of meropenem in burn patients with infections caused by gram-negative bacteria: Are we getting close to the right treatment? J Glob Antimicrob Resist 2020;20:22-27.

18. Borra LCP, Bosch TM, van Baar ME, et al. Adequacy of a hospital-wide standard dose of $7 \mathrm{mg} / \mathrm{kg}$ bodyweight gentamicin sufficient to achieve an adequate prophylactic maximum serum concentration (Cmax) in burn patients undergoing surgical burn wound treatment. Burns 2016;42: 1819-1824.

19. Lee C, Walker SAN, Walker SE, et al. A prospective study evaluating tobramycin pharmacokinetics and optimal once daily dosing in burn patients. Burns 2017;43:1766-1774.

20. Forrest A, Ballow CH, Nix DE, et al. Development of a population pharmacokinetic model and optimal sampling strategies for intravenous ciprofloxacin. Antimicrob Agent Chemother 1993;37:1065-1072.

21. Garrelets JC, Jost G, Kowalasky SF, etal. Ciprofloxacin pharmacokinetics in burn patients. Antimicrob Agent Chemother 1996;40:1153-1156.

22. Couet W, Gregoire N, Gobin P, et al. Pharmacokinetics of colistin and colistimethate sodium after a single $80-\mathrm{mg}$ intravenous dose of CMS in young healthy volunteers. Clin Pharmacol Ther 2011;89:875-879.

23. Nation RL, Li J, Cars O, et al. Framework for optimization of the clinical use of colistin and polymyxin B: The Prato polymyxin consensus. Lancet Infect Dis 2015;15:225-234.

24. Corcione S, Baietto L, Malvasio V, et al. Pharmacokinetics of colistin methanesulfonate (CMS) in burn patients. J Antimicrob Chemother 2017;72:319-321.

25. Elder K, Hill DM, Hickerson WL. Characterization of variables for potential impact on vancomycin pharmacokinetics in thermal or inhalation injury. Burns 2018;44: 658-664.

26. Rybak MJ, Le J, Lodise TP, et al. Therapeutic monitoring of vancomycin for serious methicillin-resistant Staphylococcus aureus infections: A revised consensus guideline and review by the American Society of Health-System 
Pharmacists, the Infectious Diseases Society of America, the Pediatric Infectious Diseases Society, and the Society of Infectious Diseases Pharmacists. Am J Health-Syst Pharm 2020;77:835-864.

27. Akers KS, Cota J, Chung KK, et al. Serum vancomycin levels resulting from continuous or intermittent infusion in critically ill burn patients with or without continuous renal replacement therapy. J Burn Care Res 2012;33:e254e262.

28. Lovering AM, Le Floch R, Hovsepian L, et al. Pharmacokinetic evaluation of linezolid in patients with major thermal injuries. J Antimicrob Chemother 2009;63:553559.

29. Mokline A, Gharsallah L, Rahmani I, et al. Pharmacokinetics and pharmacodynamics of linezolid in burn patients. Ann Burn Fire Disasters 2018;31:118-121.

30. Mohr JF, Ostrosky-Zeichner L, Wainright DJ, et al. Pharmacokinetic evaluation of single-dose intravenous daptomycin in patients with thermal burn injury. Antimicrob Agents Chemother 2008;52:1891-1893.

31. Murray CK, Loo F, Hospenthal DR, et al. Incidence of systemic funcal infection and related mortality following severe burns. Burns 2008;34:1108-1112.
32. Schlotman T, Akers KS. 381 Pharmacokinetics and pharmacodynamics of voriconazole in burn patients: A case series. J Burn Care Res 2018;39(Suppl 1):S161.

33. Asensio MJ, Sanchez M, Galvan B, et al. Micafungin at a standard dosage of $100 \mathrm{mg} /$ day achieves adequate plasma exposure in critically ill patients with severe burn injuries. Intensive Care Med 2015;41:371-372.

34. Neofytos D, Huang YT, Cheng K, et al. Safety and efficacy of intermittent intravenous administration of high-dose micafungin. Clin Infect Dis 2015;61(Suppl 6):S652-S661.

35. Garcia-de-Lorenzo A, Luque S, Grau S, et al. Comparative population plasma and tissue pharmacokinetics of micafungin in critically ill patients with severe burn injuries and patients with complicated intra-abdominal infection. Antimicrob Agent Chemother 2016;60:5914-5921.

Address correspondence to:

Dr. Kaitlin A. Pruskowski 3698 Chambers Pass JBSA Fort Sam Houston, TX 78234

USA

E-mail: Kaitlin.a.pruskowski.civ@mail.mil 